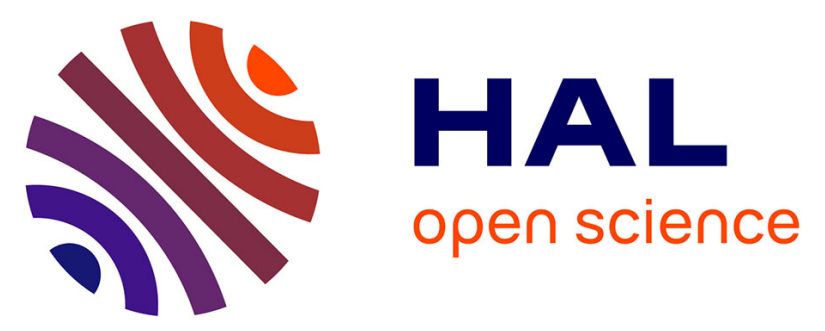

\title{
Combining qualitative evaluation and social network analysis for the study of classroom social interactions
}

\author{
Alejandra Martinez, Yannis Dimitriadis, Bartolomé Rubia-Avi, Eduardo
}

Gomez-Sanchez, Pablo de La Fuente

\section{- To cite this version:}

Alejandra Martinez, Yannis Dimitriadis, Bartolomé Rubia-Avi, Eduardo Gomez-Sanchez, Pablo de La Fuente. Combining qualitative evaluation and social network analysis for the study of classroom social interactions. Computers and Education, 2003, 41 (2003), pp.353-368. 10.1016/j.compedu.2003.06.001 . hal-00190427

\section{HAL Id: hal-00190427}

https://telearn.archives-ouvertes.fr/hal-00190427

Submitted on 23 Nov 2007

HAL is a multi-disciplinary open access archive for the deposit and dissemination of scientific research documents, whether they are published or not. The documents may come from teaching and research institutions in France or abroad, or from public or private research centers.
L'archive ouverte pluridisciplinaire HAL, est destinée au dépôt et à la diffusion de documents scientifiques de niveau recherche, publiés ou non, émanant des établissements d'enseignement et de recherche français ou étrangers, des laboratoires publics ou privés. 


\title{
Combining qualitative evaluation and social network analysis for the study of classroom social interactions
}

\author{
A. Martínez ${ }^{\text {a }}$ Y. Dimitriadis ${ }^{b}$ B. Rubia ${ }^{\text {c }}$ E. Gómez ${ }^{\text {b }}$ \\ P. de la Fuente ${ }^{\text {a }}$ \\ ${ }^{a}$ Dpt. of Computer Science, University of Valladolid, Spain \\ ${ }^{\mathrm{b}}$ Dpt. of Signal Theory, Communications and Telematics Engineering, University of \\ Valladolid, Spain \\ ${ }^{\mathrm{c}}$ Dpt. of Didactics and School Organization, University of Valladolid, Spain
}

\begin{abstract}
Studying and evaluating real experiences that promote active and collaborative learning is a crucial field in CSCL. Major issues that remain unsolved deal with the merging of qualitative and quantitative methods and data, especially in educational settings that involve both physical and computer-supported collaboration. In this paper we present a methodology that combines traditional sources of data with computer logs, and integrates social network analysis in an overall qualitative evaluation approach. Several computer tools have been developed to assist in this process, integrated with generic software for qualitative analysis. We present the method in the context of an educational and research project that has been going on for three years, to which we have incrementally applied and validated the evaluation design and tools. Our proposal and the presented case study aim at giving an answer to the need of innovative techniques for the study of new forms of interaction emerging in CSCL; at increasing the efficiency of the traditionally demanding qualitative methods, so that they can be used by teachers in curriculum-based experiences; and at the definition of a set of guidelines for bridging different data sources and methodological perspectives.
\end{abstract}

Key words: cooperative/collaborative learning, evaluation methodologies, learning communities, post-secondary education

\section{Introduction}

Design and development of Computer Supported Collaborative Learning (CSCL) systems is very complex, due to the diversity of implied actors and the variety of issues to consider: learning improvement, school organization, cultural problems, software design, distributed systems management, human-computer interaction, etc. This complexity demands appropriate methods of evaluation that let 
researchers and practitioners learn by applying innovative experiences and reflecting on them (Neale \& Carroll, 1999). The application of computers to collaborative learning has been considered as a new resource for research in the field, due to their capability of logging interactions and processing them automatically. However, it also presents new challenges, mainly related to the appearance of new collaborative situations with new forms of interaction and to problems of automatic data management and interpretation (Guribye \& Wasson, 2002).

In order to support the development of CSCL situations we proposed a conceptual framework named DELFOS ("a Description of a tele-Educational Layered Framework oriented to Learning Situations") (Osuna \& Dimitriadis, 1999). It defines an architecture for the design of CSCL applications and a development methodology based on the ideas of participatory analysis and design (Chin, Rosson, \& Carroll, 1997), which emphasizes the role of formative evaluation in the development of information systems. We are currently working on the refinement of the methods and techniques defined in DELFOS for this formative evaluation. For this purpose, we draw on a situative approach that considers both individual as well as social and participatory aspects of learning (Wilson \& Myers, 2000). In the previous version of the framework, evaluation was mainly oriented to the constructivist aspects of learning, focusing on the individual rather than on the social perspective. Therefore, we are now completing the evaluation method in DELFOS by defining a methodology and tools for evaluating social aspects related with the participation in a community of learners.

A discipline that has showed to be appropriate for the efficient study of these social and participatory aspects of learning is Social Network Analysis (SNA) (Wasserman \& Faust, 1994). Several studies have demonstrated its value within the CSCL field for the study of structural properties of individuals learning in groups (Nurmela, Lehtinen, \& Palonen, 1999; Cho, Stefanone, \& Gay, 2002). These studies usually take computer logs as an input, and process them with a SNA software package, such as UCINET (Borgatti, Everett, \& Freeman, 1999). However, SNA by itself is not enough for achieving a full understanding of the problems, and needs to be complemented with other methods, like qualitative content analysis. Unfortunately, no guidelines have been provided in the CSCL literature regarding the integration of qualitative data and methods with SNA. On the other hand, existing SNA tools require a high level of expertise and they use proprietary data formats. An approach based on XML (W3C, 2000) for the representation of collaborative interactions could then offer a means to solve the problems of heterogeneity and integration in a systematic way.

We consider that the principles of qualitative case study (Stake, 1995) constitute a good framework towards the integration of SNA methods in the evaluation of CSCL experiences. This approach draws on naturalistic research methods able to deal with the subjective and complex nature of the studied phenomenon. Case studies performed under this perspective are based on the analysis of interactions of 
the participants in the contexts where these educational actions take place. These studies use ethnographic sources of data, such as observations, questionnaires and interviews, able to capture the perceptions of the participants. Quantitative data can be used to account for the occurrence of actions or events, and relate them with the qualitative categories. This way, the quantitative data becomes an additional input in the overall qualitative approach.

In this paper we present a method that faces the new requirements posed by CSCL situations, enabling the integration of different sources of data and methods into qualitative case studies oriented to formative evaluation. Part of the data comes from event logs of computer-based tools that students use to fulfill the course assignments, while other data are collected by traditional means (formal observations, questionnaires, group interviews). As an integral part of this method we present the tools we have developed in order to increase the efficiency and usability of the evaluation procedures. In order to exemplify the discussion, we present the method in the context of the classroom-based research project named LAO (Dimitriadis, Martínez, Rubia, \& Gallego, 2001), on which we have been working during the last three years. This project has been the platform for the conception and validation of the evaluation method presented in this paper. We aim at providing answers to the need of considering new forms of interaction that arise when working with networked computers, and the need of developing an effective evaluation method able to deal with different data, methods and tools.

The rest of the paper is structured as follows: next section outlines the project to which the evaluation has been applied. Then, the research method and tools developed for its support are presented. The third section illustrates the use of the method for the study of one category extracted from the case study. We also elaborate on the advantages and limitations of the proposed methodology. Finally, we draw some conclusions and outline future lines of research.

\section{Case study description: The LAO project}

During the last three years we have been involved in a classroom-based research and development project aimed at the introduction of project-based learning with case studies and collaborative learning in a course of Computer Architecture in the studies of Telecommunications Engineering. The details of this project and its evolution can be found in (Dimitriadis et al., 2001). Here, we present its main features as they constitute the case study in which our evaluation method and tools evolved and were validated.

Following the principles of the educational model of DELFOS as well as the directives of the IEEE/ACM Computing Curricula, the project aims to provide contextualized, integrated and meaningful knowledge; promoting active, intentional and 
collaborative learning. Besides these learning objectives, the LAO project served as a platform for educational research, where several issues related to the impact of the pedagogical design and tools on attitudes towards collaboration have been studied. The experimental work has taken place during the last three years, during the fall semester (September to February) of the academic years 1999-2000 to 20012002. The general design was validated during the fist year. The revised project was extensively and systematically evaluated, in order to assess its effectiveness at fulfilling the aforementioned learning objective. General findings of this evaluation can be read in (Dimitriadis et al., 2001). Here we present the main features of the project as they constitute the setting where we have applied our evaluation design.

The whole course is defined as a project that develops along the semester, whose objective is the design and evaluation of computer systems. In order to enable distinct perspectives of the subject within the classroom, five case studies (clients) are defined, covering different market sectors and system requirements. The teacher takes the roles of the different clients and the director of the manufacturer companies. Students work in pairs, and assume the roles of a consulting firm and a computer manufacturer. Each pair is assigned one out of the five case studies for the whole course, i.e. they serve only one of the five clients. This way, in each laboratory group of at most forty students, different clients are being studied along the course. The educational design aimed at promoting interaction within and between the pairs assigned to different clients.

The project is divided into three subprojects that study different specific issues of the whole problem. Every subproject presents two milestones. In the first one, basic decisions are taken, and in the second milestone, each pair has to submit a formal technical report to the client (teacher). In every milestone, each laboratory group holds a debate, designed as a collaborative review of the work of the students, and where the problems of the different clients can be shared and discussed at a laboratory group level. At the end of the whole project, a technical report is collaboratively produced among all pairs that deal with the same case study in each laboratory group.

Several tools are used to support the project. BSCW (Basic Support for Co-operative Work) (Appelt, 1999), a well-known shared workspace system based on web interface, was used for asynchronous document sharing and threaded discussions. Of special interest here is the fact that BSCW logs every action performed on the shared workspace, providing data that were used as a source of the analysis, as explained in the following section. Other tools, like e-mail for communication and simulators for the assignments are also used.

Next section presents the method that was applied for the evaluation of the research objective of the LAO project, while specific findings in the LAO case study are presented in section 4 . 


\section{Mixed evaluation methodology for the study of social aspects of learning}

Many features included in the evaluation method proposed in this section try to give an answer to the new requirements posed by CSCL with respect to this general problem. We outline briefly these issues before proceeding with the description of the method, as they are important to understand the motivations of the proposal.

An important consequence of the use of computers to support collaborative learning is the fact that many researchers saw in it an opportunity for evaluation, due to their storage and processing capabilities. This way, log files provided by the computer are nowadays a common source of data, normally combined with more traditional ones. However, not everything is an advantage. Computer mediation of collaborative learning has introduced new challenges to evaluation. Some of them are related to new features of the situation, like the need to deal with asynchronous and distant settings (Neale \& Carroll, 1999) or new forms of interaction (Crook, 1994). Other problems refer to data management, interpretation and privacy (Guribye \& Wasson, 2002). Many studies reported in the literature use ad hoc data logs not specifically tailored for evaluation. This leads to large amounts of unnecessary and redundant data. Furthermore, the format is not suitable for direct processing, and they do not provide the semantic information needed for automatic processing. It is not common to find customization functions that allow the teacher or researcher to select the kind of study she wants to perform, or to deal with privacy issues. Therefore, challenges in CSCL research are how to make log data easily available to researchers, allowing them to configure the evaluation; how to perform data processing by automatic means; and how to present results in an intuitive format.

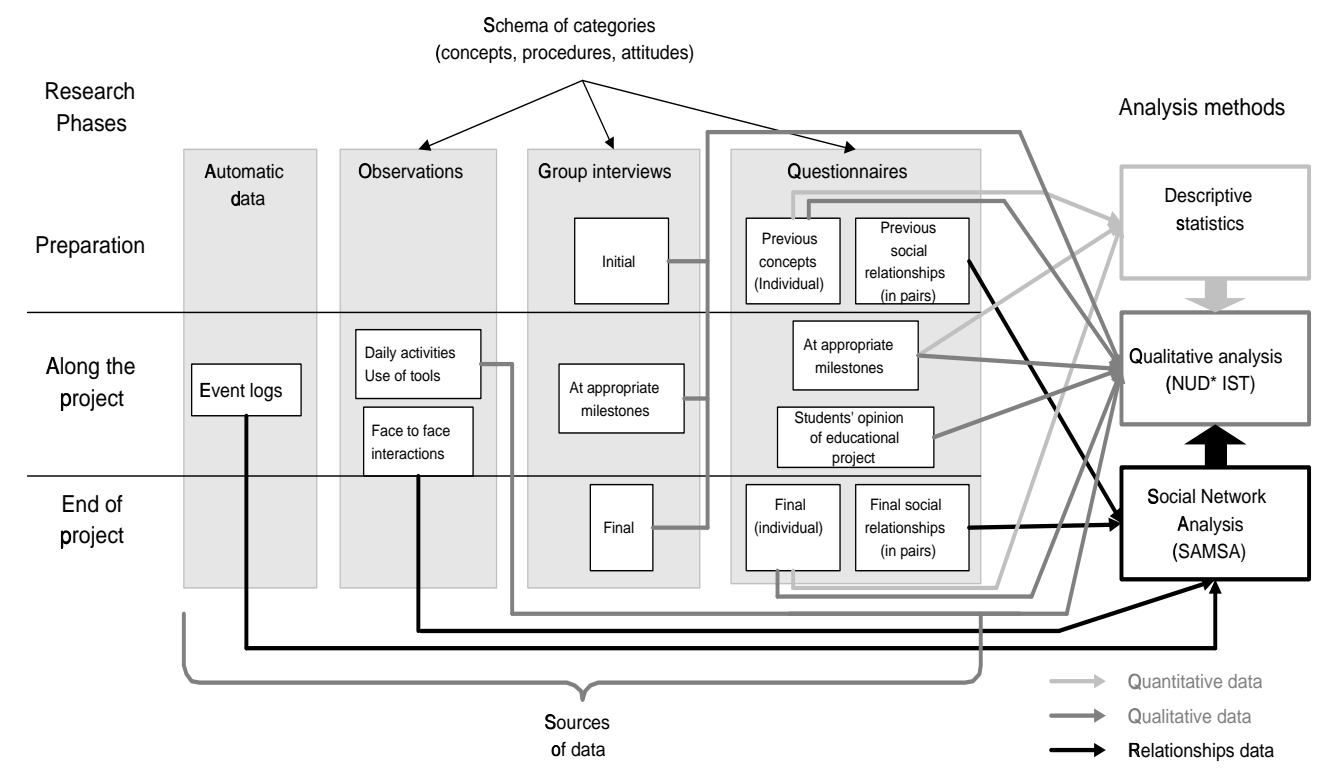

Fig. 1. The proposed mixed evaluation scheme: Data sources, methodology, timing and analysis tools. Arrows show information flow paths. 
In order to face these problems, we have defined a mixed evaluation method, depicted in figure 1. It uses several sources of data and analytical methods, and is supported by automatic tools to increase the efficiency of the overall process.

\subsection{Data sources}

The research method uses ethnographic data from a variety or sources. It combines different questionnaires: general questionnaires at the beginning and end of the course, with open and closed questions regarding the research objective; students' post hoc comments to get their short-term impressions after meaningful activities or events; students' critics about the educational project, submitted as appendices to the reports, in order to get their subjective view of the project. Group interviews are held with a group of volunteers at appropriate milestones, including the beginning and the end of the course, in order to gain insight into the students' point of view; classroom non-participant observations where an observer takes note of the different interactions and attitudes towards participation in the students' daily work at the laboratory. Finally, $\log$ files register interactions held through the CSCL tool that is being used. While interview and questionnaires are more suitable for acquiring a participant's perspective of the problem, data collected automatically and observations are better for measuring actual use of the tools and the interactions arising from it. This variety of sources aims at supporting a data triangulation scheme that considers the new requirements of CSCL settings.

Part of this data is processed by software tools, as explained in section 3.3. It is important to note that the use of these tools requires the data to be described at an appropriate level of abstraction, suitable for being processed by a computer. We have chosen XML (W3C, 2000) as the data representation format. The main reasons for this choice are self-descriptiveness, standardization, and interoperability. XML files are easy to understand and produce, as they follow a syntax that is defined by means of a DTD (Document Type Definition). In our case, this DTD describes in an abstract manner the different types of interaction that can be encountered in different CSCL scenarios. By defining this DTD, we are providing a unified representation of the distinct sources of data, and thus avoiding the cumbersome data transformation processes typical in scenarios with different data and tools. Moreover, being a generally accepted standard, developers of tools can take advantage of the increasing number of technologies based on XML; while final users (teachers, researchers) might benefit from the integration of the proposed tools with generic web technologies or another system based in XML. Further details about the DTD and its use to support evaluation can be found in (Martínez, Dimitriadis, \& de la Fuente, 2002). 


\subsection{Description of the method}

The evaluation method is based on the principles of case study research. As explained beforehand, the study of CSCL situations needs to be done from a qualitative standpoint, which aims to understand each experience considering its context and evolution. Among the qualitative methods, case studies are appropriate for evaluations, as they deal with the intensive study of one or few examples of certain phenomenon. Quantitative data can be used in the study to account for occurrences of actions and relate them with the general scheme.

As shown in figure 1, the study starts with the definition of a scheme of categories, which can be done empirically, based on the results of past experiences, or theoretically, according to the specific evaluation objectives. The scheme evolves along the study, by specialization of existing categories or addition of new ones that emerge from the analysis. This qualitative analysis is fed by qualitative data sources (open questionnaires, observations, group interviews) in a traditional manner. Additionally, quantitative data and SNA results are also submitted to the qualitative analysis, in the way explained below.

Quantitative analysis of closed questions is performed in order to account for occurrences of facts, possible problematic points, etc., in an efficient manner. It consists of simple descriptive statistical analysis assisted by any of the currently available statistical packages. We "qualitize" these quantitative data by including them as new inputs to the qualitative evaluation.

On the other hand, integration of SNA in the overall evaluation scheme is achieved by identifying a set of SNA measurements that relate to one or more of the qualitative categories. As mentioned beforehand, we have introduced SNA in the evaluation process due to its potential for the study of social roles and structural patterns related to participation in the community. Given a social network, in which actors are linked by means of one or several relationships, SNA provides structural measurements that describe the network as a whole, as well as actors' measurements, yielding information about the participation of each actor in the network. We have identified a set of SNA indicators as a starting point of our proposal: Network density $(D)$, actor's degree centrality $\left(C_{D}\left(n_{i}\right)\right)$, and network degree centralization $\left(C_{D}\right)$ (Wasserman \& Faust, 1994). $D$ measures how much knitted is a network, with values ranging from 0 (most scarce) to 1 (most dense). Degree centrality is an index of actor's prestige. Given an actor $n_{i}, C_{D}\left(n_{i}\right)$ is the proportion of actors that are adjacent to $n_{i}$. It gives an idea of the activity of the actors. In the case of directed relationships, that consider the direction of the link, two degree indexes are defined: indegree, or the number of links terminating at the node; and outdegree, or the number of links originating with the node. Finally, network degree centralization $\left(C_{D}\right)$, is a group-level measure based on actor's degree, which gives an idea about the dependency of the network on the activity of a small group of actors. Di- 
rected networks define the corresponding indexes of indegree centralization $\left(D_{I D}\right)$ and outdegree centralization $\left(D_{O D}\right)$.

A relevant feature of social networks is that they can be visualized as graphs called sociograms, which represent the actors as nodes of the graphs and the links among them as lines in the graph. A convenient representation of sociograms is produced by the use of Multidimensional Scaling (MDS). MDS maps the similarities among actors, so that those that are similar to each other in the input data appear closer in the graph, and vice versa. Using geodesic distances as a measure of dissimilarity, a sociogram will show in an intuitive manner subgroups of inter-related actors, and some relevant positions, such as the more and less prominent actors (Wasserman \& Faust, 1994).

In order to perform social network analysis, we need to define the set of networks and relationships to which the study is to be applied. This task depends on the particular context being studied. However, it is possible to propose a set of generic networks, defined from different data sources, namely: questionnaires, observations, logs of interactions through the computer. We will show examples of them in section 4.2. We should note that the use of these different data sources, as well as in the overall evaluation scheme, complements the information and provides for a more thorough and reliable understanding of the processes.

\subsection{Supporting evaluation tools}

Our global evaluation scheme is supported by three software tools: QUEST, SAMSA (System for Adjacency Matrix and Sociogram-based Analysis), and Nud*IST. Additionally, any statistical package can be used for the quantitative analysis. Figure 2 shows their use in the overall analysis method, which will be outlined in this section.

QUEST (Gómez, Dimitriadis, Rubia, \& Martínez, 2002) allows for the design of questionnaires by a teacher or researcher, and their presentation as web-based forms to the students. QUEST collects automatically the results and converts them into different formats: RTF files for its use with Nud*IST, spreadsheet files for quantitative data analysis, and XML files representing the interactions for SNA. We should point out that these automatic conversions of data are simple, but very necessary for improving the efficiency of the whole process.

SAMSA $^{1}$ supports the social network analysis procedures of the evaluation. As shown in figure 2, it contains several input modules (obs $2 \times m l$, el2 $\mathrm{xml}$ ), that take data from different sources (observations and event logs respectively) and transform them into the XML file representing the interactions. Then, SAMSA allows the re-

$\overline{1}$ Named after the subject to Kafka's Metamorphosis. 


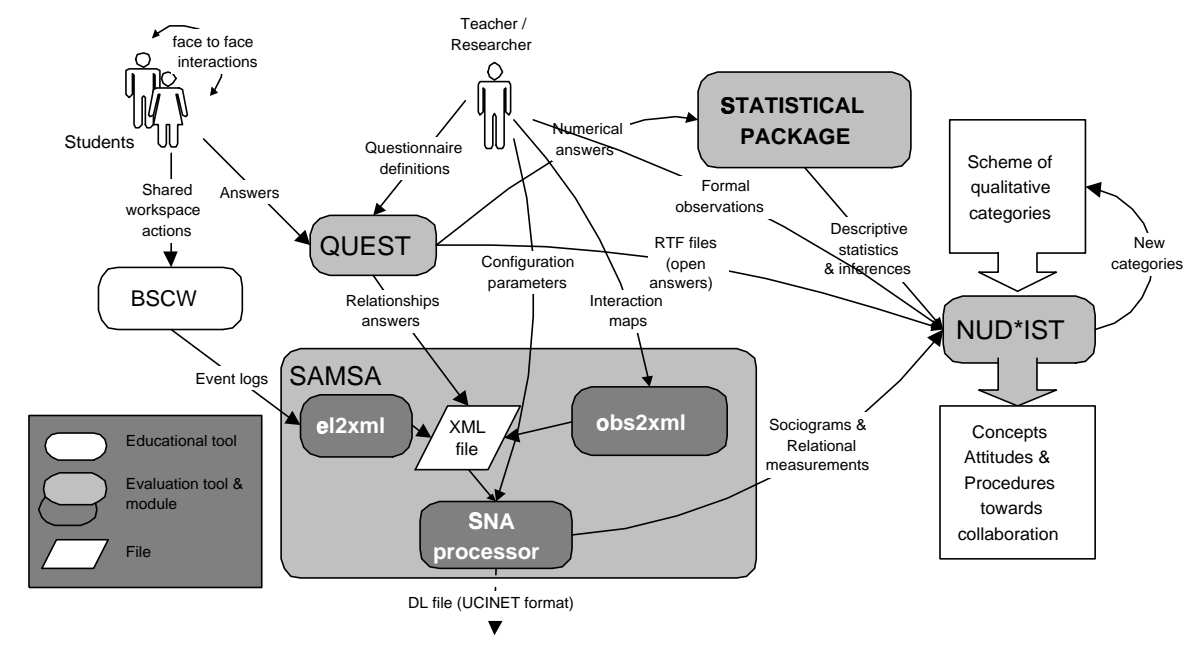

Fig. 2. The proposed analysis tools, as related to the potential actors (students, researcher, teacher), showing information flow during educational research.

searcher to select and configure the network she wants to study (selecting dates, actors, and relationship). The tool builds the matrix that represents the network, known as sociomatrix, and computes the indexes chosen by the researcher. It also shows the sociogram representing the network, and allows for actors' attributes visualization. SAMSA supports all the aforementioned SNA measurements that have been identified for our general evaluation purposes. However, deeper network analysis might be necessary to find out new features of the networks. In order to allow for this deeper analysis, SAMSA produces a DL (Data Language) file, that can be read by UCINET (Borgatti et al., 1999), a generic SNA software package. Through this feature, SAMSA supports generic social network research, by automatically converting different sources of data, and thus avoiding these costly data transformation procedures.

The overall evaluation procedure is supported by a third tool, Nud*IST (QSR, 1997), a well known qualitative data analysis package. As explained in the previous section, the qualitative analysis is at the core of the process, and therefore, this tool receives data from the rest of the elements of the system (figures 1 and 2). It takes direct input from QUEST (free-form questionnaires in RTF format), as well as the transcriptions of the observations and the group interviews. As mentioned beforehand, both the statistical analysis and the SNA results are processed with Nud*IST to fulfill the qualitative analysis.

The data sources, procedures and tools described in this section constitute our proposal of a mixed method for evaluation, in which SNA methods and quantitative statistics have been integrated in an overall qualitative scheme. SNA has been introduced due to our interest in the study of participatory aspects of learning, and quantitative data is used in order to detect general tendencies. All the methods are fed with data coming from different sources, facing the need of capturing the different forms of interaction that arise in computer-network supported classrooms. This 
way, we provide for method as well as data triangulation that will lead to increase the evaluation reliability. The need of unifying the different data sources has led us to propose the use of XML and the subsequent definition of a DTD that models different types of collaborative interaction. Next section will illustrate the use of the system with an example related to the LAO project.

\section{Collaboration as sharing information: an example of the method in the LAO project}

The method proposed in the previous section has been applied to the educational and research project described in section 2. In the present section, we illustrate the proposed scheme using a specific example, that focuses on a category (collaboration as sharing information) that is specially relevant within the context of the educational research objective of the project.

\subsection{Evaluation data and procedures of the experience}

The example presented in this section has been extracted from the general results of fall 2001 case study, that was applied to a course in the fourth (out of five) year of the studies carried out in the Telecommunications Engineering School at the University of Valladolid, Spain. The whole class of 100-120 students was divided into three sessions of forty students (maximum), in which the elementary unit consists of groups of two students (pairs). The fifteen-week long semester corresponds to three subprojects of four weeks each, where the reviews (synchronous debates) took place every two weeks. Elaboration of the final report started in the sixth week. Final report was to be submitted a month after the course had finished, and therefore, during this period, there were no lectures where students could meet face to face.

The main issue of evaluation was to know if these innovative methods would succeed in developing new concepts, attitudes and procedures towards collaboration, in the context of the passive and individualistic culture of Spanish universities. We were also interested in the role of computer-based tools in this potential change of perspective. An initial scheme of categories was defined according to these evaluation objectives, and taking into account the categories identified in fall 2000 study.

One of those categories was "collaboration as sharing information". This concept is of special interest in our educational setting for several reasons. First, the emergence of a community of learners requires the free flow of information in the group, where everybody has the feeling (and the certainty) that they can access important data available in the community. This was a major challenge in our project, as the previous experience of the students was closer to that found in traditional 
classrooms, where information is expected to flow mainly between teacher and students. Second, computer supported collaboration in the project takes place mainly through sharing information: BSCW can be used in order to share reports, papers, URLs and other information resources, and students were strongly encouraged to do so by the teacher. And finally, the educational project was also designed in order to promote these sharing interactions. The students were asked to write the final technical report among all the pairs of each laboratory group dealing with the same client, thus requiring them to share (at least) their subproject reports. They were also asked to compare their solutions with the rest of the clients, which was itself a means of encouraging the students to access other's pairs information.

The evaluation followed the scheme presented in section 3, adapted to the particular characteristics of the project. Formal observations took place every week in one of the laboratory groups, and group interviews were held with a group of ten volunteers. Questionnaires were submitted to the students following the evaluation design, and BSCW events were logged during the whole course. Several social networks were constructed to inspect the interactions. The example presented in this section aims at showing how the different sources of data, methods and techniques of analysis provide a better understanding of the concept under study.

\subsection{Analysis procedures}

Right before the course started, previous concepts related to collaboration were inspected through two questionnaires, one devoted to establish an initial social network of collaboration, while the other wondered for their perception of it. The results of the former were processed with SAMSA, yielding the indexes shown in the first column of table 1 . They indicate that their previous experiences did not include sharing information as a main form of collaboration (this concept is the least dense, with $D_{i}=0.29 \%$ ), whereas solving doubts and creating a product in common were the most dense relationships. In addition, in the latter questionnaire only a few students regarded the classroom environment as collaborative (16.5\%), and some others $(30.6 \%)$ said that collaboration happened only with friends. Surprisingly, most pairs (75 vs. 10) declare themselves motivated to work in group. This points out an apparent contradiction: they appreciate collaboration as an abstract value, but their previous experience is rather poor and does not actually tend towards collaborative attitudes.

Our evaluation setting allows us to observe the evolution of this concept, since the same two questionnaires were repeated right after the course finished. Regarding the relationships questionnaire, the density of all activities increased significantly, notably sharing of information, that almost doubles its value. Positive results also appeared in the final general questionnaire, where the same question about the classroom collaboration environment got the following distribution: $6.8 \%$ very 
competitive; $18.2 \%$ competitive; $9.1 \%$ indifference; $40.9 \%$ only with friends. This initial observation reflects the positive impact of the educational project, but should be further confirmed by the rest of the analysis tools in our methodology.

Table 1

Density measurements from the networks built from questionnaires $\left(D_{i}\right.$ : initial questionnaire network density; $D_{f}$ : final questionnaire network density)

\begin{tabular}{lrr}
\hline Relationship & $D_{i}$ & $D_{f}$ \\
\hline Discussing & $0.34 \%$ & $0.48 \%$ \\
\hline Solving doubts & $0.43 \%$ & $1.11 \%$ \\
\hline Sharing information & $0.29 \%$ & $0.53 \%$ \\
\hline Create a product in common & $0.77 \%$ & $0.82 \%$ \\
\hline
\end{tabular}

With respect to the means for sharing information, BSCW offers a shared workspace where students were encouraged to publish their documents, pieces of information, and notes, while the teacher used it to deliver documents and comments to the class. An obvious question that emerges now is whether BSCW helped the aforementioned development of collaborative attitudes with respect to sharing information. In order to answer this question, a social network was constructed on the basis of indirect relationships that stem from posting and reading others people's documents, so that a link between $n_{i}$ and $n_{j}$ represents $n_{j}$ accessing an object created by $n_{i}$. The two networks, one including the teacher and other leaving him out, will allow to test his overall influence in the use of BSCW. Table 2 shows density and centralization indexes of the networks, divided in four periods: the three subprojects ( $\mathrm{sp1}$, sp2, sp3) and the final project (spf). The two columns to the left show results of the network including the teacher, while columns to the right, results without him.

Both networks show that density decreases along time, during the three subprojects. The low use of BSCW in this period of the course was confirmed by mid-course, through a questionnaire performed in one of the milestones (post-review questionnaire), in which students were asked if they intended to post their notes and intermediate documents in BSCW, and a latter questionnaire asking if they actually did so and why. Surprisingly, twenty pairs expressed their intention of sharing their documents, but just three of them did it. When asked in the following milestone through an open-ended questionnaire about the reasons of this contradiction, they argued lack of time or confidence in their contributions.

Nevertheless, the table shows a sharp peak of density in the period of the final project. This increase of activity is explained by several reasons: at this time of the year they were on examination period without lectures, and therefore, they could not meet face to face. They started to post drafts, notes, comments, and numerical results in BSCW and read almost all the documents posted by other pairs having the same client (i.e. the same case study), which supports the idea that collaboration as sharing information develops partially influenced by the relationship of 
collaboration as generating a common product.

On the other hand, outdegree centralization $\left(C_{O D}\right)$ gives an idea of how balanced was the creation of documents, which can be regarded as active sharing of information in BSCW. By observing the results it is possible to see that the teacher has a strong influence, with a peak in the second subproject (sp2). By inspecting the XML translation of the BSCW logs, we could easily find out that during this period, the teacher published articles and comments regarding the first subproject reports, that were read by all the students. $C_{O D}$ is also very high in the final project (spf), when the teacher published comments to the successive versions of the final reports submitted by the students. These comments were massively read by the students.

Table 2

Indexes from the BSCW indirect interactions network. D: normalized density; $C_{O D}$ : outdegree centralization

\begin{tabular}{l|rr|rr}
\hline & \multicolumn{3}{|l|}{ Without teacher } & \multicolumn{3}{|c}{ With teacher } \\
\hline Period & $D$ & $C_{O D}$ & $D$ & $C_{O D}$ \\
\hline Sp1 & $17.65 \%$ & $115.57 \%$ & $21.93 \%$ & $374.38 \%$ \\
\hline Sp2 & $13.73 \%$ & $88.93 \%$ & $17.54 \%$ & $1668.21 \%$ \\
\hline Sp3 & $10.13 \%$ & $137.02 \%$ & $14.33 \%$ & $226.85 \%$ \\
\hline Spf & $31.05 \%$ & $157.79 \%$ & $35.38 \%$ & $775.31 \%$ \\
\hline
\end{tabular}

Sociograms complement the information of the tables above, showing at first glance an idea of how the different actors (pairs, in this case), are situated with respect to the relationship. Figure 3 shows the sociograms of the indirect relationships network during the fourth period of the course, corresponding to the final project writing, with the teacher (x00) included in the network. We can observe several interesting features. First, the network shows a complete graph (all nodes are connected to each other), which was not the case with other networks built from the questionnaires and from the maps of interactions taken in the observations. Therefore, a first conclusion is that BSCW succeed at eliminating obstacles between the students. Second, we see that, in spite of the teacher being a most central actor as explained beforehand, some pairs $(x 26, x 34, x 23, \times 39)$ occupy central positions in the sociogram. Inspecting the logs, we could see that some of these pairs had published notes and comments along the course, which were massively read by the rest of the students. Thus, these central students could be identified as being the information "sharers" of the class. Third, pairs are placed nearby others that share the same client, due to the fact that in this period BSCW was used mainly to exchange information and messages related to the final report (that had to be written among several pairs with the same client). Since this client-centered organization cannot be observed in the sociograms corresponding to the rest of the subprojects (not shown here), we may conclude again that writing a joint report (i.e. having a common product) significantly increased collaboration through sharing information. This is confirmed by the following statements from the final group interview: "[...] in this 
project we did use BSCW quite a lot to make appointments, to publish the things, to..., in this one we did, in the others we almost did not"; "We used BSCW so that the person in charge of putting the pieces together, could do it at home in a moment, and then the next day we met with the printed version and on it we were modifying it."

Concluding this section, we can resume some of the findings that refer to the evaluation objective and point out how the proposed scheme helped to achieve the evaluation goal.

Results yielded by the quantitative analysis of the pre- and post-course questionnaires reflected an improvement in the student's perception of their own collaborative attitudes towards sharing information. This positive result was partially confirmed by the rest of the methods, which helped to uncover some new aspects. Comparing the differences between the social networks of the three subprojects and the final one, we can conclude that BSCW was mainly used to get information from the teacher, and only in the last period students started to share information through it. A deeper qualitative analysis of student's answers to open-ended questions, and of the group interviews confirmed that this increase in the interchange of information was mainly due to practical reasons, and not so much to the aim of sharing their own knowledge. On the other hand, quantitative analysis showed that the general perception of the classroom collaborative environment improved dramatically after the course. In conclusion, we can say that the educational design almost obliged the students to practice new forms of collaboration that, in spite of not being as constructive as intended, helped to improve the overall classroom collaborative environment.

Regarding the evaluation method itself, we have also observed that sociograms can intuitively unveil interaction patterns, both through the computer (using computer $\operatorname{logs}$ ) or directly (using the observer notes as source data). The discovered patterns can be confirmed by density and centralization scores, provided by the SNA tools. When it comes to explain more, pure qualitative tools like Nud*IST help categorize the information coming from the qualitative data sources, as questionnnaires, interviews or the observer annotations. Therefore, the combination of several sources of data results extremely convenient to avoid false or incomplete conclusions. However, we have seen that triangulation in mixed evaluation schemes may impose an unaffordable burden on researchers, in terms of time efficiency and usability. Our experience at the LAO project can suggest that the proposed tools together with the guidelines may significantly alleviate this problem in CSCL systems evaluation. 


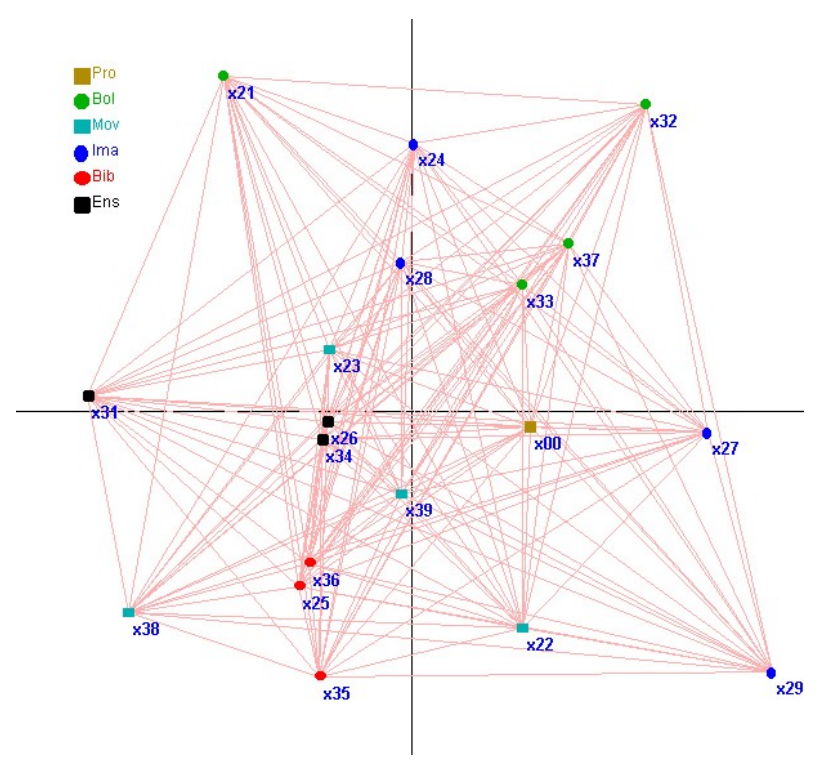

Fig. 3. Sociogram of the indirect relationships through BSCW network during the last period (writing of the final report). The teacher and the different clients are represented in different styles.

\section{Conclusions and future work}

We have proposed a mixed evaluation method that aims at the study of participatory aspects of CSCL environments, by including SNA techniques, quantitative statistics, and computer data logs into an overall qualitative case study design. The proposed scheme has been extensively tested during a three year period in a semipresential university environment with considerable success and its main observations have been presented in this paper. We can argue that the proposed evaluation design is general enough, and its ideas can be adopted in CSCL environments different from the one that has been considered. This statement has been validated by our own experience in the evaluation of a significantly different environment based on a synchronous collaborative puzzle for kindergarten children (Martínez et al., 2002).

One of the major educational research issues tackled by our design refers to usability and time efficiency. When working on formative evaluation, time limits are very important, as corrective actions could be of no value if results come too late. In this sense, QUEST proved very efficient and flexible at designing, collecting and converting questionnaire data into adequate formats (both qualitative and quantitative). Besides that, SAMSA allows for an easy and efficient processing of data coming from various sources (interaction maps from observations, computer logs, questionnaires) and provides configurable and easy to visualize SNA results. The enhancement in usability and efficiency has been clearly observed along the three years of field work within the LAO project. As the proposed method, guidelines and tools evolved, our effort could mainly focus on the evaluation objectives, be- 
ing also able to react with corrective actions and to support partially the formative evaluation process of the course teacher.

The use of XML to represent the interactions provides for the conceptual and operational integration of the different data under a common description. It is the core of a loosely coupled architecture, in which new modules can be added to deal with new sources of data. Additionally, developers can take advantage of the large offer of XML-based technologies found in the market. All these facts are increasingly important if we consider that a multifaceted evaluation scheme requires the use of multiple data sources, and that CSCL settings tend to be composed by a number of different and independent tools.

Several issues need still to be addressed. One of the most important refers to the fact that triangulation depends heavily on the expertise of the researcher/teacher. A deep knowledge of the context, a precise and careful design of the research objectives and categories, as well as a good use of the tools are required for a successful approach. Although several of these problems are clearly related to naturalistic evaluation approaches in general, we aim at providing a more refined set of guidelines as well as tools based on techniques of Artificial Intelligence.

Ongoing research deals with the integration of this perspective with the existing constructivist evaluation of DELFOS, which will allow us to reflect on the integration of individual and social aspects of learning. This could lead to consider new SNA techniques related to the roles of actors and their positions within the network.

\section{Acknowledgements}

The authors would like to acknowledge the contributions of Olga Velloso, Judit Tardajos, Inmaculada Garrachón and Jose María Marcos (development of SAMSA), Raquel Díaz and M. José Gallego (setup of the initial experimental environment), Dr. César Osuna (DELFOS framework), the rest of the transdisciplinary group EMIC, and last but not least all the students that eagerly participated in these academic experiences. Partial financial support for this research project was given by the Autonomous Government of Castilla and León, Spain (VA18/99, VA117/01), the Ministry of Science and Technology, Spain and the European Funds for Regional Development (TIC2000-1054) and the European Union Leonardo Programme.

\section{References}

Appelt, W. (1999). WWW Based Collaboration with the BSCW System. Proceedings of SOFSEM'99, Springer Lecture Notes in Computer Science 1725 (p. 
66-78). Milovy.

Borgatti, S., Everett, M., \& Freeman, L. (Eds.). (1999). UCINET 5.0 Version 1.00. Natick: Analytic Technologies.

Chin, G., Rosson, M., \& Carroll, J. (1997). Participatory analysis: Shared development of requirements from scenarios. Proceedings of Human Factors in Computing Systems, CHI'97 Conference (p. 162-197). N.Y.: ACM.

Cho, H., Stefanone, M., \& Gay, G. (2002). Social Information Sharing in a CSCL Community. Proceedings of Computer Support for Collaborative Learning, CSCL 2002 (p. 43-50). Boulder, CO.

Crook, C. (1994). Computers and the collaborative experience of learning. London: Routeledge.

Dimitriadis, Y., Martínez, A., Rubia, B., \& Gallego, M. (2001). Cooperative Learning in Computer Architecture: an educational project and its telematic support. Proceedings of the Conference of Frontiers in Education, FIE 2001. Reno, NE.

W3C (2000). Extensible markup language (XML).(http: //www.w3.org/XML/)

Gómez, E., Dimitriadis, Y., Rubia, B., \& Martínez, A. (2002). Quest, a telematic tool for automatic management of student questionnaires in educational research. Proceedings of $2^{\text {nd }}$ European Conference on Technology, Information, Education and Citizenship, TIEC 2002. Barcelona.

Guribye, F., \& Wasson, B. (2002). The ethnography of distributed collaborative learning. Proceedings of Computer Support for Collaborative Learning, CSCL 2002 (pp. 637-638). Boulder, CO.

Martínez, A., Dimitriadis, Y., \& de la Fuente, P. (2002). CSCL formative evaluation support by means of analysis of interactions (in Spanish). Proceedings of IV Simposio Internacional de Informática Educativa, IE 2002. Vigo. (To appear)

Neale, D., \& Carroll, J. (1999). Multi-faceted evaluation of complex, distributed activities. Proceedings of Computer Support for Collaborative Learning, CSCL'99. Stanford, Palo Alto, CA.

Nurmela, K., Lehtinen, E., \& Palonen, T. (1999). Evaluating CSCL log files by Social Network Analysis. Proceedings of Computer Support for Collaborative Learning, CSCL'99. Stanford, Palo Alto, CA.

Osuna, C., \& Dimitriadis, Y. (1999). A Framework for the Development of Educational Collaborative Applications based on Social Constructivism. Proceedings of CYTED RITOS International Workshop on Groupware, CRIWG '99 (p. 71-80). Cancún, Quintana Roo.

QSR (1997). NUD*IST. Software for qualitative data analysis. Thousand Oaks, CA: Scolari.

Stake, R. (Ed.). (1995). The art of case study research. Thousand Oaks, CA: SAGE Publications, Inc.

Wasserman, S., \& Faust, K. (1994). Social Network Analysis: Methods and Applications. Cambridge: Cambridge University Press.

Wilson, B., \& Myers, K. (2000). Situated Cognition in Theoretical and Practical Context. In D. Jonassen \& S. Land (Eds.), Theoretical foundations of learn- 
ing environments (pp. 57-88). Mahwah, N.J.: Lawrence Erlbaum Associates, publishers. 